STRUCTURAL BIOLOGY

ISSN 2059-7983

Keywords: editorial; structural biology

\section{Expanding beyond biological crystallography}

\author{
Jennifer L. Martin, ${ }^{\mathrm{a} *}$ Randy J. Read ${ }^{\mathrm{b} *}$ and Soichi Wakatsuki ${ }^{\mathrm{c} *}$
}

anstitute for Molecular Bioscience, University of Queensland, Queensland Bioscience Precinct, Brisbane, QLD 4072, Australia, ${ }^{\mathbf{b} C a m b r i d g e}$ Institute for Medical Research, Department of Haematology, University of Cambridge, Wellcome Trust/MRC Building, Hills Road, Cambridge CB2 OXY, United Kingdom, and 'Photon Science, SLAC and Structural Biology, School of Medicine, Stanford University, 2575 Sand Hill Road, \#MS 69, Menlo Park, CA 94025, USA.

*Correspondence e-mail: j.martin@imb.uq.edu.au, rjr27@cam.ac.uk, soichi.wakatsuki@stanford.edu

We are witnessing a revolution in structural biology. Electron microscopy, XFELs, and hybrid structural approaches have transformed the field, creating new ways to understand biological molecules, and how they operate. Meanwhile, the acceleration of growth in Protein Data Bank entries continues unabated. In 2015, the number of entries surged to more than 114000 . That's 100000 more than in 1999. The boom in biological structures and the new ways we can probe them have led us at Acta $D$ to update our mission and scope.

Biological crystallographers don't just use biological crystallography any more. They have become structural biologists who choose the best structural method, or combination of methods, to address the fundamental biological questions they ask. Their toolbox now includes methods like single-particle cryoEM, NMR, chemical cross-linking, SAXS/ SANS, molecular dynamics simulations, mass spectrometry, EPR and fluorescence techniques, but this is by no means an exhaustive list. Moreover, with the expansion of the databases, the structure of a biological molecule on its own now rarely provides sufficient new insight for understanding function. Mutational studies, cellular studies and biochemical analyses often enrich the structural and functional landscape that better enables us to comprehend the workings of biological machines.

These factors led your Acta $D$ Section Editors to convene in person and electronically in 2015 to re-evaluate our mission and to draw up a new scope for the articles we wish to attract to the journal. Our over-arching aim is to ensure Acta D continues to be the preeminent biological crystallography journal of the IUCr. We aspire to be the journal where all structural biologists, not just biological crystallographers, send their best papers. Consequently, we have widened the journal scope to encourage submission of papers that include biological crystallography or other structural biological methods when combined with functional data, where the combination of the structural and functional data reveal significant new insight. Of course we continue to welcome methodology papers, a mainstay of our journal since its inception in 1993.

The new subtitle we have selected for Acta Crystallographica Section D, Structural Biology reflects this change in scope. Acta $D$ still focuses on papers that present significant new insights into any of the biological, biochemical, or medical sciences. On the other hand, articles that report a biological structure without supporting functional data are not generally suitable for submission to Acta D. Acta F, our sister journal, will continue to be the home for the rapid publication of structural biology papers, including high quality protein crystal structures. With these changes, the names of the two journals are now more closely aligned - Acta Cryst. D, Structural Biology focuses on high impact structural biology research and Acta Cryst. F, Structural Biology Communications emphasizes the rapid publication of important structural biology research.

The closeness of our two structural biology journals is also shown in another change for 2016; after over 12 years as a Section Editor for Acta D, Zbyszek Dauter is moving to become one of the Section Editors of Acta F. We would like to thank Zbyszek for all his work on Acta $D$ and wish him well in his new role at Acta F.

We are very excited by the prospects of widening the journal scope to welcome a diversity of structural biology papers, and we look forward to receiving submissions that highlight novel and impactful biological structure, function and methods. 